\title{
Ultrasound study of dynamic behaviour of left ventricle in genetic asymmetric septal hypertrophy
}

\author{
F. J. TEN CATE, P. G. HUGENHOLTZ, AND J. ROELANDT \\ From the Department of Clinical Echocardiography, Thoraxcenter, University Hospital Dijkzigt and Erasmus \\ University, Rotterdam, the Netherlands
}

Genetic asymmetric septal hypertrophy is a primary myocardial disease with characteristic echocardiographic features. Echocardiographic studies in patients have shown that the ventricular septum is a hypocontractile structure. Since overall cardiac systolic function in this disease is normal, the present study was undertaken to explain this phenomenon.

Nineteen patients with proven genetic asymmetric septal hypertrophy, and 15 patients with coronary artery disease and echocardiographic akinesis of the ventricular septum with an angiographically complete obstruction of the descending branch of the left coronary artery, were compared with 20 normal subjects.

Analysis includes calculation of systolic thickening of the septum and left ventricular posterior wall, calculation of systolic posterior wall velocity, and determination of left ventricular internal dimensions with ultrasound.

Results showed that the ventricular septum was a hypocontractile structure in asymmetric septal hypertrophy, whereas the left ventricular posterior wall seems to compensate for this as is seen by the augmented indices for systolic thickening and velocity of the posterior wall. Left ventricular size was small. This was in contrast to the findings in coronary artery disease with septal akinesia. Indices for thickening and velocity of the posterior wall were decreased, whereas left ventricular size was enlarged. We propose that systolic function of the left ventricle can be maintained by three different compensatory mechanisms; (1) increase of dimension (Starling mechanism); (2) increase in systolic thickening; and (3) increase in systolic velocity of contraction.

Genetic asymmetric hypertrophy of the interventricular septum is a primary myocardial disease with distinct morphological abnormalities (Henry et al., 1973).

Several studies using electron microscopy have identified ultrastructural changes consisting of abnormal architecture of the interventricular septum with bizarrely shaped muscle cells (Ferrans et al., 1972; Maron et al., 1974).

Although one would expect these changes to result in an impaired overall cardiac function, this does not occur. Indeed, the systolic cardiac function in these patients is often normal (Goodwin and Oakley, 1972). In an attempt to clarify this enigma, an echocardiographic study was undertaken. In this study we paid special attention to the posterior wall of the left ventricle and analysed its contraction pattern in an effort to explain the existence of a normal overall cardiac systolic function in this disease.

Received for publication 9 August 1976

\section{Patients and methods}

Nineteen patients with genetic asymmetric septal hypertrophy, 11 women and 8 men, ages 14 to 53 years were studied.

The diagnosis was based on the echocardiogram (septal hypertrophy was defined as a thickness greater than $15 \mathrm{~mm}$, and a ratio of septal to posterior wall thickness of greater than $2: 1$ ).

All patients underwent haemodynamic evaluation and left ventriculography. In 11 patients, a resting gradient across the left ventricular outflow tract was present and all patients had abnormalities consistent with asymmetric hypertrophy on their right anterior oblique left ventricular cine angiocardiogram. In addition, 15 patients with coronary artery disease, all having an angiographically complete obstruction of the descending branch of the left coronary artery and septal akinesia on their echocardiograms, were also studied.

As a control group 20 'normal' volunteers were 


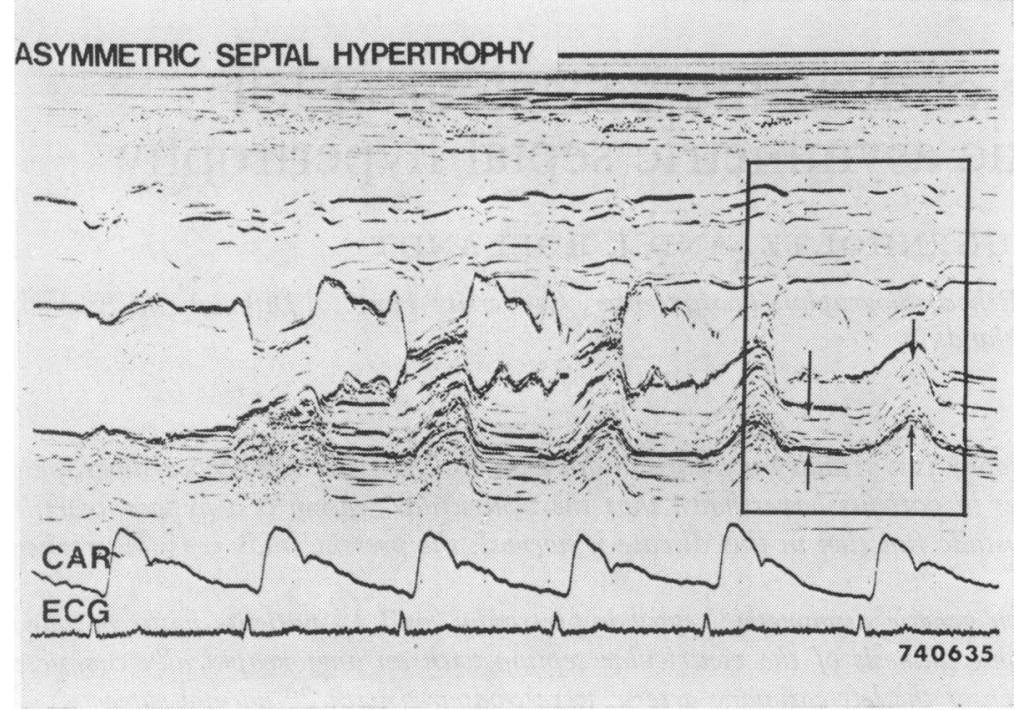

Fig. 1 A standard M-mode sectorscan in a patient with haemodynamic proof of genetic asymmetric septal hypertrophy. The motion pattern of the anterior mitral leaflet is normal during systole, indicating no outflow obstruction. The area studied is that part of the left ventricle between the tips of the mitral valves and the papillary muscles. This is shown at the right of the picture. The two important features are that: (1) the motion of the interventricular septum is much reduced as is the systolic thickening and (2) the motion pattern of the left ventricular wall is increased and shows excessive thickening. used. ('Normal' was defined on the basis of the absence of complaints, clinical examination of the heart, electrocardiogram, and chest $x$-ray).

\section{ECHOCARDIOGRAPHIC EXAMINATION}

The ECHOcardioVISOR 01 (Organon Teknika, Oss, The Netherlands), which has both the conventional single and the recently developed twodimensional imaging facilities was used (Kloster et al., 1973; Roelandt et al., 1974).

A $1.4 \mathrm{~cm}, 2.25 \mathrm{MHz}$ transducer focused at $7.5 \mathrm{~cm}$ was employed and the $M$-mode echocardiograms recorded simultaneously with the electrocardiogram by a Honeywell Linescan-recorder (Visicorder 1856) on light-sensitive paper (Kodak Linagraph 1895). All patients were examined in the supine or slightly left lateral recumbent position, with the transducer placed as close as possible to the left side of the sternum and perpendicular on the chest wall until the characteristic pattern of motion of the anterior mitral valve leaflet was maximally recorded. The ultrasonic beam was then directed laterally and slightly inferiorly to define the 'standard area' of the left ventricle just distal to the tips of the mitral valve in which the motion of the interventricular septum and left ventricular posterior wall could be detected together. After the standard left ventricular echocardiograms, sector scans were made and recorded from the aortic root across the left ventricular cavity to the left ventricular apex. During the examination gain settings were continuously adjusted until the best available recordings could be made.

Fig. 1 shows a representative $M$-mode sector scan of a patient with genetic asymmetric septal hypertrophy without left ventricular outflow gradient under basal conditions during cardiac catheterisation. The disproportionate thickness of the interventricular septum compared with the left ventricular posterior wall thickness is striking. Furthermore, the motion of the interventricular septum is reduced compared with the increased motion of the left ventricular posterior wall.

In order to show the accuracy of the single element recordings another procedure was carried out. It is evident that a single beam recording does not show motion perpendicular to the sound beam axis
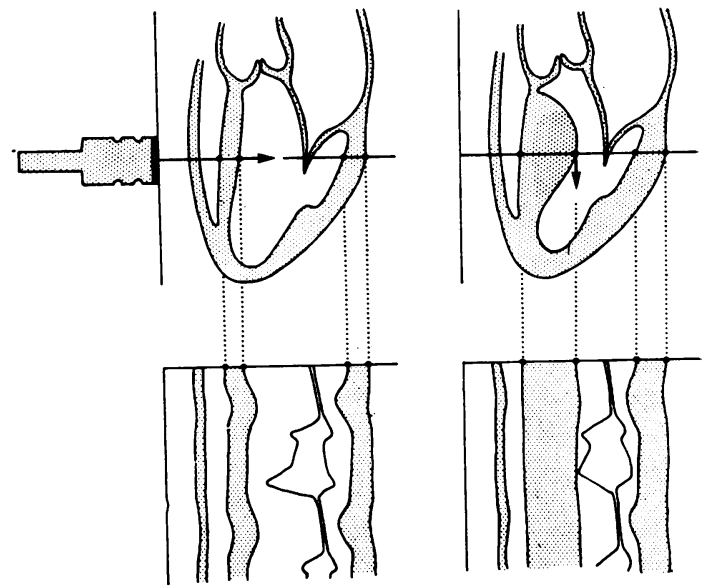

Fig. 2 Motion of cardiac structures as recorded in $M$-mode (left side) is not seen when this motion is perpendicular to the sound beam (right side). 


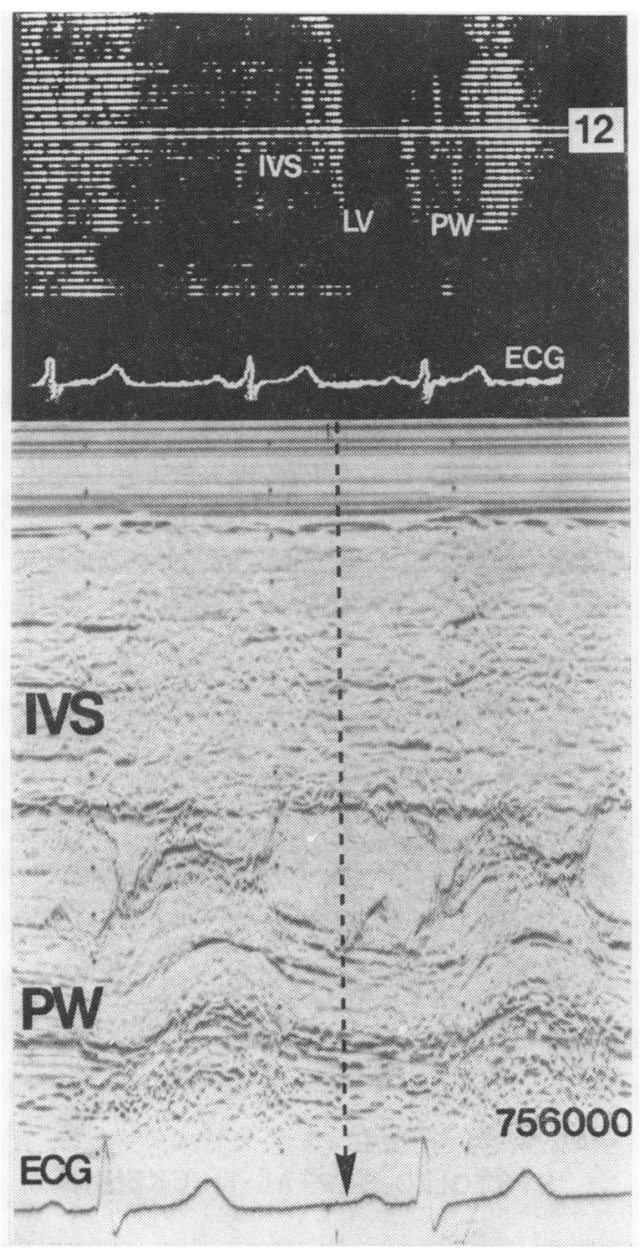

Fig. 3 Stop-frame image in diastole obtained from a patient with outflow obstruction. A single crystal of the 20 crystal transducer at the level of the anterior mitral valve leaflet-labelled 12-is selected and its echo information is recorded in the $M$-mode and represented below. The dotted line shows the timing of the cross-sectional image within the cardiac cycle.

(Fig. 2). Therefore, real time two-dimensional imaging was performed to observe this motion. This was done by selection of a single crystal out of the 20-crystal transducer at the level of the tip of the anterior mitral leaflet. A recording of this echo information in the M-mode is shown in Fig. 3.

This procedure was repeated using two elements -one above and one below the chosen one-in order to show that septal thickness is not changed by a vertical motion which could be seen from the twodimensional real time images (Fig. 4). This procedure was included in the echocardiographic examination of all patients studied.

\section{ANALYSIS OF ECHOCARDIOGRAPHIC DATA}

The left ventricular internal diameter (D) was measured in $\mathrm{mm}$ from the left septal endocardium to the left ventricular posterior wall endocardium at end-diastole (Dd) and at end-systole (Ds) (enddiastole at the R-peak of the QRS complex and endsystole at the shortest dimension (ten Cate et al., 1974)).

Septal and posterior wall thickness $(T)$ was also measured at the same points of reference $(\mathrm{Td}=$ enddiastole and $T s=$ end-systole). Fractional or per cent systolic thickening of both interventricular septum and left ventricular posterior wall was determined as $\frac{\mathrm{Ts}-\mathrm{Td}}{\mathrm{Td}} \times 100 \%$ (in $\%$ ) where $\mathrm{Ts}$ and $\mathrm{Td}$ are the systolic and diastolic thickness of either the interventricular septum or left ventricular posterior wall. Normalised posterior wall velocity was calculated as $\frac{\mathrm{Ts}-\mathrm{Td}}{\mathrm{Td} \times \mathrm{ET}}$, where ET is the ejection time measured from the onset to peak contraction (Cooper et al., 1972) (see also Fig. 8).

Student's $t$ test for independent observation was used to determine the statistical significance of these measurements (Fisher, 1970).

\section{Results}

The results of our measurements are summarised in the Table. Systolic thickening of the interventricular septum in the 3 groups of patients studied is shown in Fig. 5. Differences between the normal group and the patients with genetic asymmetric septal hypertrophy, as well as those with coronary artery disease, were statistically significant $(\mathrm{P}<0.005)$.

Table Results of echocardiographic measurements $(m \pm S D)$

\begin{tabular}{|c|c|c|c|c|c|c|}
\hline & $\begin{array}{l}\text { Heart rate } \\
\text { (beats/ } \\
\text { min) }\end{array}$ & $\begin{array}{l}\text { IVS } \\
\text { thickening } \\
\text { (\%) }\end{array}$ & $\begin{array}{l}\text { LVPW } \\
\text { thickening } \\
\text { (\%) }\end{array}$ & $\begin{array}{l}\text { Normalised } \\
P W \\
\text { velocity }\end{array}$ & $\underset{(\mathrm{mm})}{D d}$ & $\begin{array}{l}D s \\
(\mathrm{~mm})\end{array}$ \\
\hline $\begin{array}{l}\mathbf{N} \\
\text { ASH } \\
\text { CAD }\end{array}$ & $\begin{array}{l}72 \pm 6 \\
77 \pm 7 \\
70 \pm 8\end{array}$ & $\begin{array}{l}42 \pm 8^{\star} \\
10 \pm 6^{\star} \\
15 \pm 12^{\star}\end{array}$ & $\begin{array}{l}52 \pm 9^{\star} \\
70 \pm 30^{\star} \\
33 \pm 8^{\star}\end{array}$ & $\begin{array}{l}1.2 \pm 0.2^{\star} \\
1.7 \pm 0.5^{\star} \\
1.3 \pm 0.4\end{array}$ & $\begin{array}{l}53 \pm 5^{\star} \\
41 \pm 10^{\star} \\
63 \pm 11^{\star}\end{array}$ & $\begin{array}{l}38 \pm 4^{\star} \\
26 \pm 7^{\star} \\
50 \pm 13\end{array}$ \\
\hline
\end{tabular}

$\mathrm{N}$, normal subjects; ASH, asymmetric septal hypertrophy; CAD, coronary artery disease; IVS interventricular septum; LVPW, left ventricular posterior wall; $P$ W, posterior wall. $\star_{\mathrm{P}}<0.005$.

Fig. 6 shows a typical example of a patient with asymmetric septal hypertrophy with a posterior wall systolic thickening of 100 per cent. In Fig. 7, the results of systolic thickening of the left ventri- 


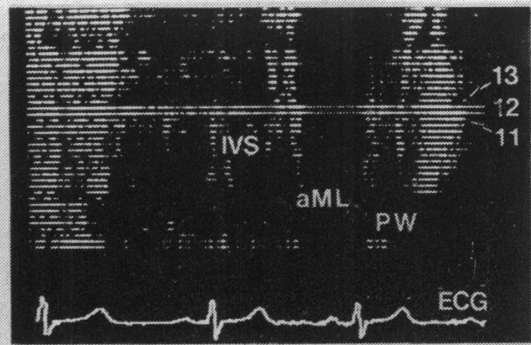

II

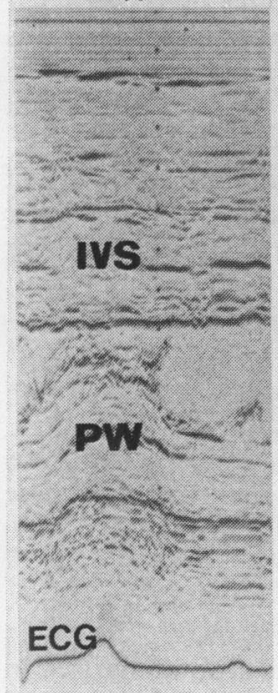

12

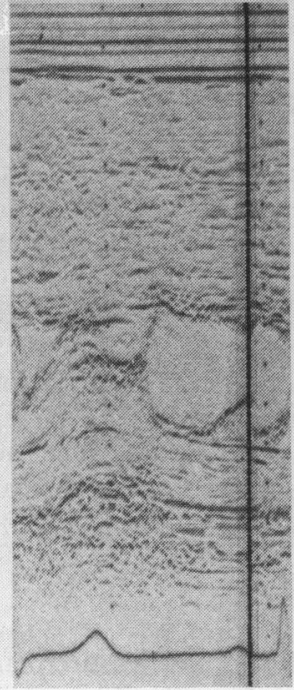

13

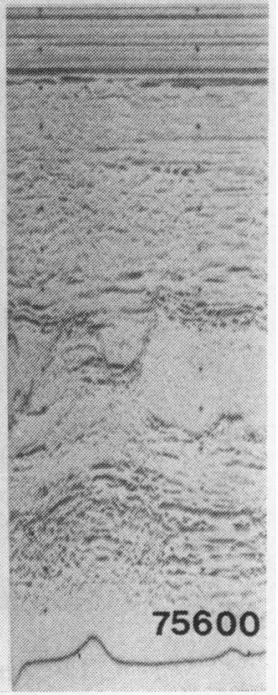

Fig. 4 The procedure described for crystal 12 in Fig. 3 is repeated for crystal 11 and crystal 13. M-mode recording of these 3 consecutive elements shows no large difference in systolic thickening of the septum or the posterior ventricular wall, indicating that vertical motion seen on the real-time two-dimensional images does not influence quantitative measurements by single element echocardiography.
Fig. 5 Systolic septal thickening in 20 normal subjects is $42 \pm 8$ per cent, $10 \pm 6$ per cent in 19 patients with genetic asymmetric septal hypertrophy, and $15 \pm 12$ per cent in 15 patients with coronary artery disease and septal akinesia. The difference between systolic septal thickening in normals and in patients with asymmetric septal hypertrophy or coronary artery disease is statistically significant $(P<0.005)$ (values expressed as mean $\pm 2 S D$ ).
SYSTOLIC SEPTAL THICKENING

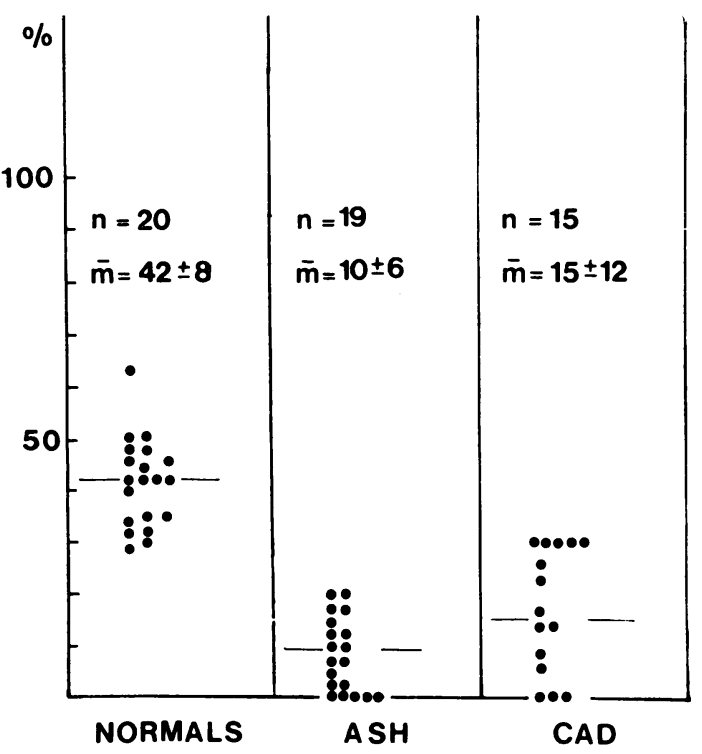


cular posterior wall for the same groups of patients are summarised. Values of left ventricular posterior wall thickening were significantly higher $(P<0.005)$ in asymmetric septal hypertrophy as compared with normals, whereas these values were significantly lower in patients with coronary artery disease and septal akinesia $(P<0.005)$. Fig. 8 shows the normalised posterior wall velocity of $2.6 \mathrm{~s}^{-1}$ in a representative patient with genetic asymmetric septal hypertrophy. Fig. 9 shows the values for the normalised posterior wall velocity in the three patient groups studied.

Again values for left ventricular posterior wall normalised velocity were significantly higher in asymmetric septal hypertrophy $(P<0.005)$, whereas no statistical significance has been found $(P<0 \cdot 10)$ between patients with coronary artery disease and normals.

The Table also shows that left ventricular internal dimensions in asymmetric septal hypertrophy both in diastole and systole were definitely smaller $(P<0.005)$ than in the normal group, whereas in patients with coronary artery disease and septal akinesia these dimensions were higher than in normal individuals $(P<0.005)$.

\section{Discussion}

Most patients with genetic asymmetric septal hypertrophy display a clinically observable rapid arterial pulse pressure rise corresponding to a rapid systolic pressure rise in the left ventricle and an increased ejection fraction. These observations suggest an overall left ventricular systolic function which is better than normal. However, echocardiographic analysis of septal contraction (Rossen et al., 1974; Cohen et al., 1975) and the morphological findings of cardiac muscle in this disease (Ferrans et al., 1972) suggest that at least this part of the left ventricle has a reduced function. In this study, the mechanical behaviour of the left ventricle in genetic asymmetric septal hypertrophy was studied by analysis of the contraction patterns of the interventricular septum and left ventricular posterior

Fig. 7 Systolic posterior wall thickening in 20 normal individuals is $52 \pm 9$ per cent, in 19 patients with asymmetric septal hypertrophy $70 \pm 30$ per cent, and in the 15 patients with coronary artery disease $33 \pm 8$ per cent. Systolic posterior wall thickening in normal individuals and in patients with coronary artery disease is significantly different from that seen in patients with asymmetric septal hypertrophy $(P<0.005)$. Note that patients with asymmetric septal hypertrophy have a large range of systolic thickening (values expressed as mean $\pm 2 S D$ ). wall, including the calculation of systolic thickening and the 'normalised' wall velocity. The results indicate that in genetic asymmetric septal hypertrophy the interventricular septum is indeed a hypocontractile structure with decreased values for

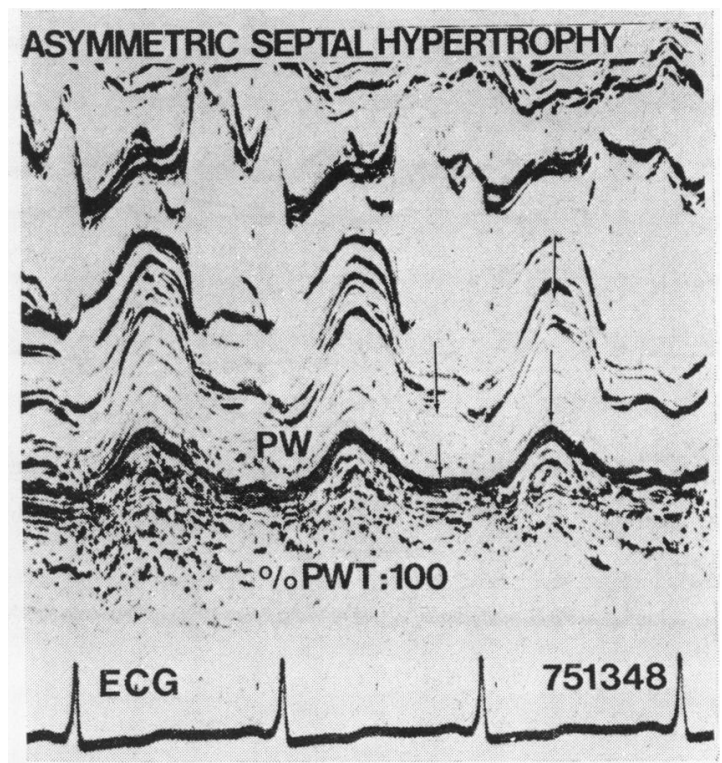

Fig. 6 Representative example of the posterior wall dynamics in a patient with asymmetric septal hypertrophy. Systolic posterior wall thickening in this particular case is 100 per cent.

\section{SYSTOLIC POSTERIOR WALL THICKENING}

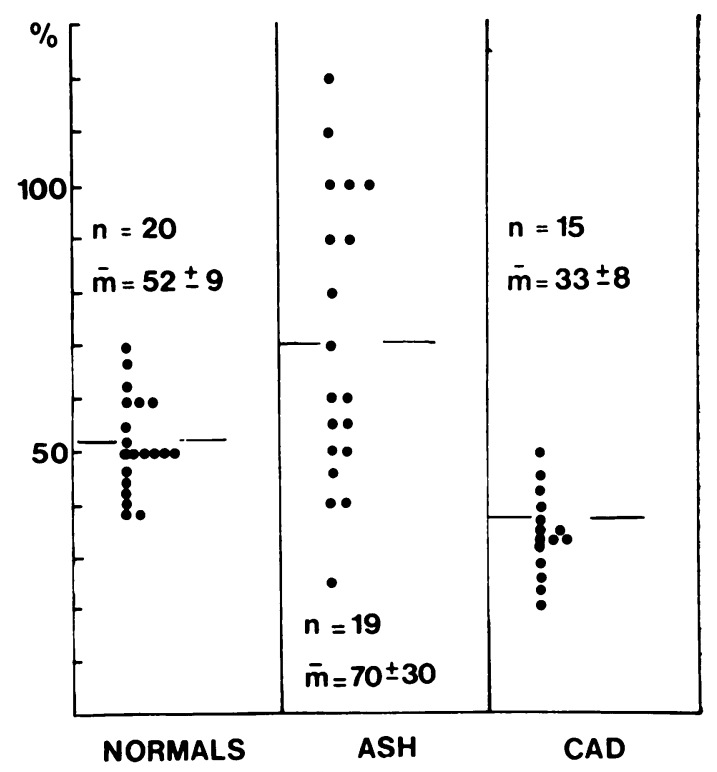




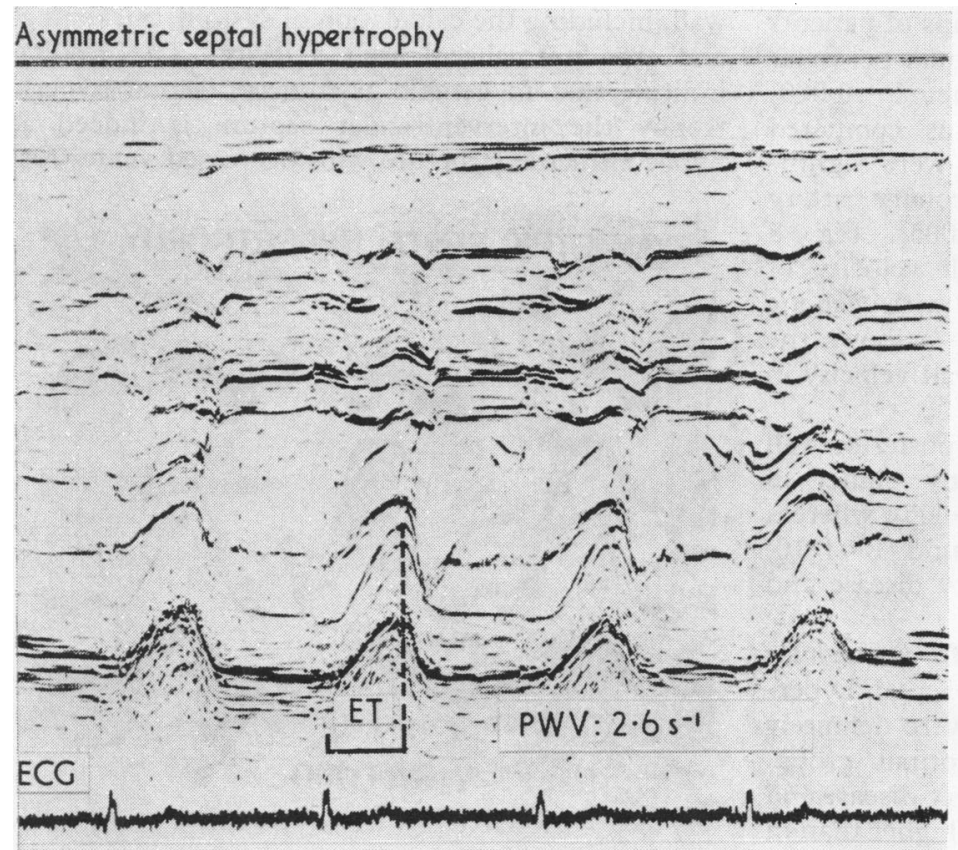

Fig. 8 Representative example of normalised posterior wall velocity in a patient with asymmetric septal hypertrophy ( $A S H)$. Ejection time is calculated as the time from peak QRS to the point where the internal dimension of the left ventricle is smallest. $50 \mathrm{~ms}$ is subtracted from this value to account for the isovolumic phase (Cooper et al., 1972). The value of normalised posterior wall velocity in this particular patient with asymmetric septal hypertrophy is $2 \cdot 6 s^{-1}$.

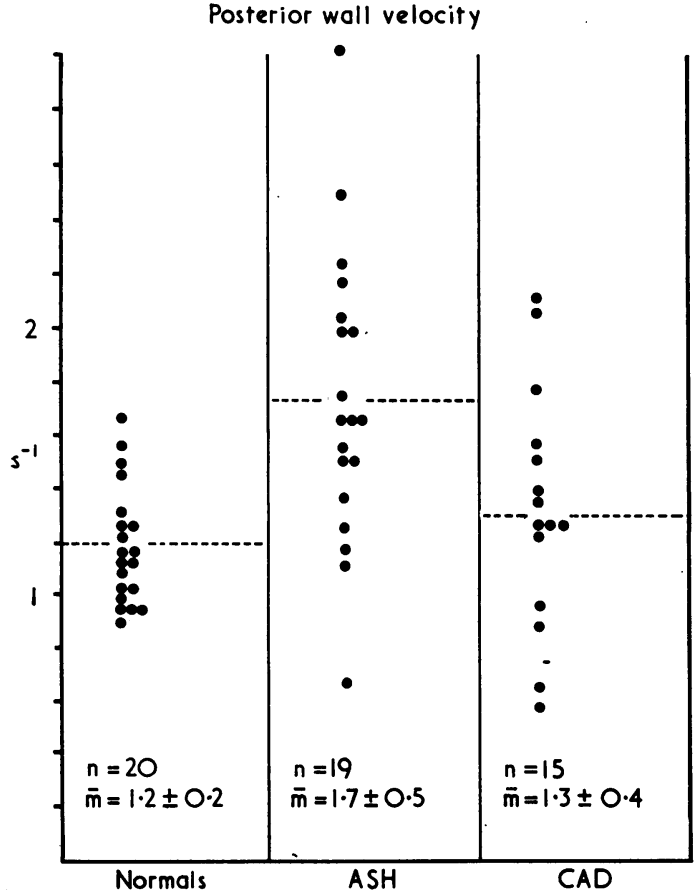

Fig. 9 Normalised posterior wall velocity in 20 normal individuals was $1 \cdot 2 \pm 0.2 \mathrm{~s}^{-1}$, for 19 patients with asymmetric septal hypertrophy (ASH) $1 \cdot 7 \pm 0.5 s^{-1}$, and for 15 patients with coronary artery disease (CAD) $1 \cdot 3 \pm 0.4 s^{-1}$. Normalised posterior wall velocity is statistically significantly different for patients with asymmetric septal hypertrophy compared with normals $(P<0.005)$, whereas this is not the case for patients with coronary artery disease $(P<0 \cdot 10)$. Note that in the three groups studied there is a broad range of values for normalised posterior wall velocity (values are expressed as mean $\pm 2 S D)$. 


\section{COMPENSATORY MECHANISMS}

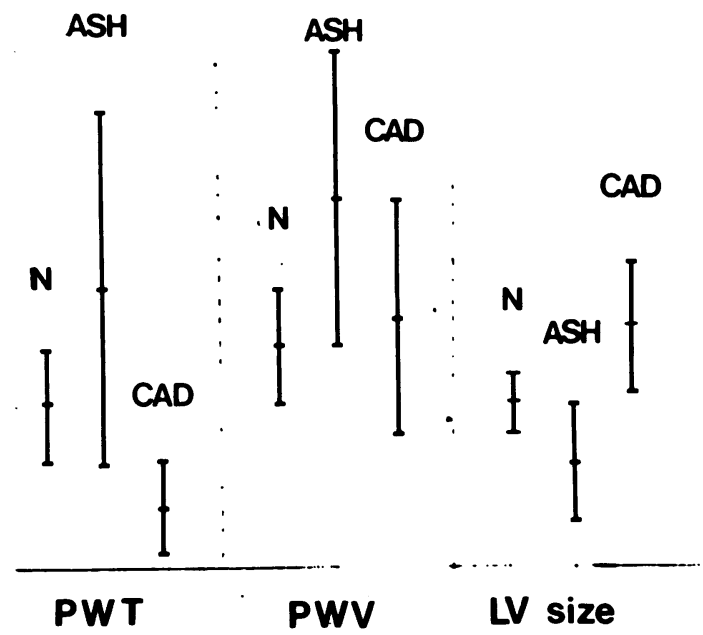

Fig. 10 Left ventricular compensatory mechanisms in the three groups of individuals studied. Patients with asymmetric septal hypertrophy ( $A S H$ ) compensate for the akinetic interventricular septum by a greater posterior wall thickening ( $P W T$ ) and higher velocity of contraction of the posterior wall $(P W V)$, whereas left ventricular size remains small, as compared with normal subjects. This is not true for patients who have coronary artery disease with septal akinesia. In these patients posterior wall thickening and velocity of contraction are not augmented, while left ventricular size is usually greater than normal.

systolic thickening and that the left ventricular posterior wall tends to compensate for this effect. Indeed both systolic thickening and contraction velocity are higher than the corresponding indices measured from normal individuals. However, this is not the only mechanism that the left ventricle has available to maintain its overall systolic function, which becomes apparent when patients with coronary artery disease and septal akinesia are analysed. In those patients the left ventricular posterior wall percentage systolic thickening was uniformly decreased whereas the contraction velocity was normal. However, in these patients the left ventricular dimensions were larger than in the asymmetric septal hypertrophy group (Fig. 10). Thus it appears that the left ventricle has three different compensatory mechanisms in order to maintain its systolic function: (1) increase dimension (Starling mechanism); (2) increase systolic thickening, and (3) increase velocity of systolic contraction.

A review of the data reveals that the interventricular septum in genetic asymmetric septal hypertrophy is indeed a hypocontractile structure. Con- tractile function of left ventricular posterior wall as assessed from its systolic thickening and velocity is augmented or at least normal. Thus, the left ventricular mechanical behaviour in genetic asymmetric septal hypertrophy is mainly determined by normal or augmented left ventricular posterior wall contraction pattern while left ventricular size remains small. This is in contrast to findings in patients with coronary artery disease, where the left ventricular dimensions are increased while systolic thickening and normalised posterior wall velocity are usually decreased. These findings suggest that the left ventricle has at least three different compensatory mechanisms to maintain its systolic function as noted above.

\section{References}

Cohen, M. V., Cooperman, L. B., and Rosenblum, R. (1975). Regional myocardial function in idiopathic hypertrophic subaortic stenosis. An echocardiographic study. Circulation, 52, 842-847.

Cooper, R. H., O'Rourke, R. A., Karliner, J. S., Peterson, K. L., and Leopold, G. R. (1972). Comparison of ultrasound and cineangiographic measurements of the mean rate of circumferential fiber shortening in man. Circulation, 46, 914-923.

Ferrans, V. J., Morrow, A. G., and Roberts, W. C. (1972). Myocardial ultrastructure in idiopathic hypertrophic subaortic stenosis. A study of operatively excised left ventricular outflow tract muscle in 14 patients. Circulation, 45, 769792.

Fisher, R. A. (1970). Statistical Methods for Research Workers, 14th ed. Oliver and Boyd, Edinburgh.

Goodwin, J. F., and Oakley, C. M. (1972). The cardiomyopathies. British Heart fournal, 34, 545-552.

Henry, W. L., Clark, C. E., and Epstein, S. E. (1973) Asymmetric septal hypertrophy: echocardiographic identification of the pathognomonic anatomic abnormality of IHSS. Circulation, 47, 225-233.

Kloster, F. E., Roelandt, J., ten Cate, F. J., Bom, N., and Hugenholtz, P. G. (1973). Multiscan echocardiography. II. Technique and initial clinical results. Circulation, 48, 1075-1084.

Maron, B. J., Ferrans, V. J., Henry, W. L., Clark, C. E., Redwood, D. R., Roberts, W. C., Morrow, A. G., and Epstein, S. E. (1974). Differences in distribution of myocardial abnormalities in patients with obstructive and nonobstructive asymmetric septal hypertrophy (ASH). Circulation, 50, 436-446.

Roelandt, J., Kloster, F. E., ten Cate, F. J., van Dorp, W. G., Honkoop, J., Bom, N., and Hugenholtz, P. G. (1974). Multidimensional echocardiography. An appraisal of its clinical usefulness. British Heart fournal, 36, 29-43.

Rossen, R. M., Goodman, D. J., Ingham, R. E., and Popp, R. L. (1974). Ventricular systolic septal thickening and excursion in idiopathic hypertrophic subaortic stenosis. New England Fournal of Medicine, 291, 1317-1319.

ten Cate, F. J., Kloster, F. E., van Dorp, W. G., Meester, G. T., and Roelandt, J. (1974). Dimensions and volumes of left atrium and ventricle determined by single beam echocardiography. British Heart fournal, 36, 737-746.

Requests for reprints to Dr. F. J. ten Cate, Department of Clinical Echocardiography, Thoraxcenter, P.O. Box 1738, Rotterdam, the Netherlands. 